\title{
A nearly exact method for solving large-scale TRS
}

\author{
M.S. Apostolopoulou, D.G. Sotiropoulos, C.A. Botsaris and P. Pintelas
}

\begin{abstract}
We present a matrix-free method for the large scale trust region subproblem (TRS), assuming that the approximate Hessian is updated using a minimal-memory BFGS method, where the initial matrix is a scaled identity matrix. We propose a variant of the Moré-Sorensen method that exploits the eigenstructure of the approximate Hessian, and incorporates both the standard and the hard case. The eigenvalues and the corresponding eigenvectors are expressed analytically, and hence a direction of negative curvature can be computed immediately. The most important merit of the proposed method is that it completely avoids the factorization, and the trust region subproblem can be solved by performing a sequence of inner products and vector summations. Numerical results are also presented.

Index Terms-Trust region subproblem, nearly exact method, L-BFGS method, eigenvalues, negative curvature direction, large scale optimization
\end{abstract}

\section{INTRODUCTION}

We consider the following quadratic minimization problem:

$$
\min _{d \in \mathbb{R}^{n}} \phi(d)=g^{T} d+\frac{1}{2} d^{T} B d, \quad \text { s.t. } \quad\|d\|_{2} \leq \Delta,
$$

where $B$ is a $n \times n$ real symmetric (possibly indefinite) matrix, $g \in \mathbb{R}^{n}, \Delta$ is a positive scalar, and $d$ is the real unknown $n$-vector. Problem (1) arises in many applications as: forming subproblems for constrained programming [2], [3], regularization methods for ill-posed problems [11], graph partitioning problems [7], large-scale nonlinear multicommodity flow problems [15], image restoration [17], etc. In particular, problem (1) is important in a class of methods for solving both convex and nonconvex nonlinear optimization problems, namely, the trust-region algorithms [3]. At each iteration $x_{k}$ of a trust-region algorithm, a trial step $d_{k}$ is usually obtained by solving the quadratic subproblem (1) where $\phi_{k}(d)$ is an approximation to the objective function $f, g_{k}=\nabla f\left(x_{k}\right), B_{k} \in \mathbb{R}^{n \times n}$ is either the Hessian or a (positive definite or indefinite) approximate Hessian of $f$ at $x_{k}$, and $\Delta_{k}>0$ is the trust region radius.

Various methods for calculating approximate solutions of TRS have been developed such as the dogleg method [16], the two-dimensional subspace minimization methods [18] and the truncated CG methods [6], [20]. Nearly exact methods for solving (1) have been proposed by Gay [5], Sorensen [19], and Moré and Sorensen [12]. The method of nearly exact solutions uses Newton's method to find a root of a scalar function that is almost linear on the interval

M. S. Apostolopoulou is with the Department of Mathematics, University of Patras, Patras, Greece. E-mail: msa@math.upatras.gr

D. G. Sotiropoulos is with the Department of Informatics, Ionian University, Corfu, Greece. E-mail: dgs@ionio.gr

C. A. Botsaris is with the Department of Regional Economic Development,University of Central Greece, Levadia, Greece. E-mail: botsaris@otenet.gr

P. Pintelas is with the Department of Mathematics, University of Patras, Patras, Greece. E-mail: pintelas@math.upatras.gr of interest. It is based on the Cholesky factorization for solving a linear system of the form $(B+\lambda I) d=-g$, where $I \in \mathbb{R}^{n \times n}$ is the identity matrix, $\lambda \geq 0$ is the Lagrange multiplier, and $B+\lambda I$ positive semi-definite. Moreover, in the so called hard case, a direction of negative curvature is required to be produced [12]. Therefore, this method can be very costly and even prohibitively expensive when it is applied in very large problems.

In this work we are concentrated in the method of nearly exact solutions.We study the eigenstructure of minimalmemory BFGS matrices, and apply our results for the solution of large scale subproblems. The proposed nearly exact method avoids the Cholesky factorization for the solution of the linear system $(B+\lambda I) d=-g$, while a direction of negative curvature is produced using the method of inverse iteration [9].

\section{Properties of the Minimal-MEMory BFGS MATRICES}

The minimal memory BFGS matrices [10], [13] are updated using the BFGS formula

$$
B_{k+1}=B_{k}-\frac{B_{k} s_{k} s_{k}^{T} B_{k}}{s_{k}^{T} B_{k} s_{k}}+\frac{y_{k} y_{k}^{T}}{s_{k}^{T} y_{k}},
$$

where $s_{k}=x_{k+1}-x_{k}$ and $y_{k}=g_{k+1}-g_{k}$, storing curvature information from the most previous iteration. We consider as the initial matrix $B_{k}^{(0)}$, the diagonal matrix $B_{k}^{(0)}=\theta_{k} I$, where $\theta_{k} \in \mathbb{R} \backslash\{0\}$, and the resulting minimal memory BFGS scheme takes the form

$$
B_{k+1}=\theta_{k+1} I-\theta_{k+1} \frac{s_{k} s_{k}^{T}}{s_{k}^{T} s_{k}}+\frac{y_{k} y_{k}^{T}}{s_{k}^{T} y_{k}} .
$$

Note that in the quadratic model (1), the approximate Hessian matrix can be positive definite or indefinite. Hence, for the remaining of the paper we only assume that $\|B\|$ is bounded, that is, there is a positive constant $M$, such that $\left\|B_{k}\right\| \leq M<\infty$, for all $k$.

Theorem II.1: Suppose that one update is applied to the symmetric matrix $B^{(0)}=\theta I, \theta \in \mathbb{R} \backslash\{0\}$, using the vector pair $\left\{s_{k}, y_{k}\right\}$ and the BFGS formula. The characteristic polynomial of the symmetric matrix $B_{k+1} \in \mathbb{R}^{n \times n}$, defined in (3), has the general form

$$
p(\lambda)=\left(\lambda-\theta_{k+1}\right)^{n-2}\left(\lambda^{2}-\beta_{1} \lambda+\beta_{2}\right),
$$

where $\beta_{1}=\theta_{k+1}+y_{k}^{T} y_{k} / s_{k}^{T} y_{k}$, and $\beta_{2}=\theta_{k+1} s_{k}^{T} y_{k} / s_{k}^{T} s_{k}$. Moreover, if the vectors $s_{k}$ and $y_{k}$ are linearly independent then the smallest eigenvalue of $B_{k+1}$ is distinct.

Proof: First we show that $B_{k+1}$ has at most two distinct eigenvalues. To this end, we consider the matrix $\bar{B}=\theta_{k+1} I-\theta_{k+1} s_{k} s_{k}^{T} / s_{k}^{T} s_{k}$ with rank $(n-1)$. Applying the interlacing theorem [22, pp. $94-98]$ on $\bar{B}$, it is easy to 
see that $\bar{B}$ besides the zero eigenvalue, has one more eigenvalue equals to $\theta_{k+1}$, of multiplicity $(n-1)$. If $B_{k+1}^{(0)}$ is positive definite, then the addition of the term $y_{k} y_{k}^{T} / s_{k}^{T} y_{k}$ on $\bar{B}$ yields

$$
\lambda_{n} \geq \theta_{k+1} \geq \lambda_{n-1} \geq \theta_{k+1} \geq \ldots \geq \theta_{k+1} \geq \lambda_{1} \geq 0,
$$

where $\lambda_{i}, i=1, \ldots, n$ denote the eigenvalues of $B_{k+1}$. The above relation implies that

$$
\lambda_{2}=\ldots=\lambda_{n-1}=\theta_{k+1} \quad \text { and } \quad \lambda_{n} \geq \theta_{k+1} \geq \lambda_{1} .
$$

Suppose now that $B_{k+1}$ is indefinite. Then, if $\theta_{k+1}>0$, from the interlacing theorem we have that

$$
\theta_{k+1} \geq \lambda_{n} \geq \theta_{k+1} \geq \lambda_{n-1} \geq \ldots \geq \theta_{k+1} \geq \lambda_{2} \geq 0 \geq \lambda_{1},
$$

which imply that

$$
\lambda_{3}=\ldots=\lambda_{n}=\theta_{k+1} \text { and } \theta_{k+1} \geq \lambda_{2} \geq \lambda_{1} .
$$

In different case $\left(\theta_{k+1}<0\right)$ we yield

$$
0 \geq \lambda_{n} \geq \theta_{k+1} \geq \lambda_{n-1} \geq \theta_{k+1} \geq \ldots \geq \theta_{k+1} \geq \lambda_{1} .
$$

Consequently,

$$
\lambda_{2}=\ldots=\lambda_{n-1}=\theta_{k+1} \quad \text { and } \quad \lambda_{n} \geq \theta_{k+1} \geq \lambda_{1} .
$$

In all the above cases, it is obvious that $B_{k+1}$ has at most two distinct eigenvalues and one eigenvalue equals to $\theta_{k+1}$ of multiplicity at least $(n-2)$. Denoting by $\lambda_{x}$ and $\lambda_{y}$ the two unknown distinct eigenvalues, the characteristic polynomial of $B_{k+1}$ can be written as follows:

$$
p(\lambda)=\left(\lambda-\theta_{k+1}\right)^{n-2}\left[\lambda^{2}-\left(\lambda_{x}+\lambda_{y}\right) \lambda+\lambda_{x} \lambda_{y}\right] .
$$

Taking into account that

$$
\operatorname{tr}\left(B_{k+1}\right)=\sum_{i=1}^{n} \lambda_{i}=(n-2) \theta_{k+1}+\lambda_{x}+\lambda_{y}
$$

and

$$
\operatorname{det}\left(B_{k+1}\right)=\prod_{i=1}^{n} \lambda_{i}=\theta_{k+1}^{n-2} \lambda_{x} \lambda_{y}
$$

we have that

$$
\begin{aligned}
p(\lambda)= & \left(\lambda-\theta_{k+1}\right)^{n-2} \\
& \left\{\lambda^{2}-\left[\operatorname{tr}\left(B_{k+1}\right)-(n-2) \theta_{k+1}\right] \lambda+\operatorname{det}\left(B_{k+1}\right) / \theta_{k+1}^{n-2}\right\} .
\end{aligned}
$$

Using the well-known properties of the trace and determinant of matrices, we yield:

$$
\operatorname{tr}\left(B_{k+1}\right)=(n-1) \theta_{k+1}+\frac{y_{k}^{T} y_{k}}{s_{k}^{T} y_{k}}
$$

and

$$
\operatorname{det}\left(B_{k+1}\right)=\theta_{k+1}^{n-1} \frac{s_{k}^{T} y_{k}}{s_{k}^{T} s_{k}}
$$

Hence, $\operatorname{tr}\left(B_{k+1}\right)-(n-2) \theta_{k+1}=\theta_{k+1}+\frac{y_{k}^{T} y_{k}}{s_{k}^{T} y_{k}}, \frac{\operatorname{det}\left(B_{k+1}\right)}{\theta_{k+1}^{n-2}}=$ $\theta_{k+1} \frac{s_{k}^{T} y_{k}}{s_{k}^{T} s_{k}}$, and relation (4) follows immediately.

It remains to show that when $s_{k}$ and $y_{k}$ are linearly independent, the smallest eigenvalue is distinct. Suppose that the vectors $s_{k}$ and $y_{k}$ are linearly independent and assume that $B_{k+1}$ has at most one distinct eigenvalue, which implies that either $\lambda_{x}=\theta_{k+1}$ or $\lambda_{y}=\theta_{k+1}$. Combining relations (8), (10), and (9), (11), we have that $\left(s_{k}^{T} y_{k}\right)^{2}=s_{k}^{T} s_{k} y_{k}^{T} y_{k} \Rightarrow \cos \phi= \pm 1$, where $\phi$ denotes the angle of $s_{k}$ and $y_{k}$. This implies that the vectors $s_{k}$ and $y_{k}$ are collinear, which contradicts the hypothesis. Hence, if the vectors are linearly independent, then $B_{k+1}$ has exactly two distinct eigenvalues. Combining relations (5), (6) and (7), easily we can conclude that $\lambda_{1}$ is always distinct.

If the vectors $s_{k}$ and $y_{k}$ are collinear, i.e., $y_{k}=\kappa s_{k}$, $\kappa \in \mathbb{R}$, then $B_{k+1}$ becomes

$$
B_{k+1}=\theta_{k+1} I+\left(\kappa-\theta_{k+1}\right) \frac{s_{k} s_{k}^{T}}{s_{k}^{T} s_{k}} .
$$

Based on Theorem II.1, the eigenvalues of $B_{k+1}$ sorted into increasing order are

$$
\kappa=\lambda_{1} \leq \lambda_{2}=\lambda_{3}=\ldots=\lambda_{n}=\theta_{k+1},
$$

and $p(\lambda)=\left(\lambda-\theta_{k+1}\right)^{n-1}(\lambda-\kappa)$. Easily can be verified that in this case the eigenvector corresponding to $\lambda_{1}=\kappa$ equals $s_{k}$, while the generalized eigenvectors corresponding to $\theta_{k+1}$ are $u_{i}=\left(-s_{k}^{i} / s_{k}^{1}, 0, \ldots, 1,0, \ldots, 0\right)^{T}$, where $i=2, \ldots, n$ denotes the $i$ th component of $s_{k}$, and 1 is in the $i$ th row of $u_{i}$.

When $s_{k}$ and $y_{k}$ are linearly independent, for being able to determine the eigenvectors corresponding to distinct eigenvalues of $B_{k+1}$, we can make use of the inverse power method [9]. Given a non-zero starting vector $u^{(0)}$, inverse iteration generates a sequence of vectors $u^{(i)}$, generated recursively by the formula

$$
u^{(i)}=(B-\hat{\lambda} I)^{-1} \frac{u^{(i-1)}}{\left\|u^{(i-1)}\right\|},
$$

$i \geq 1$, where $\hat{\lambda}=\lambda+\epsilon, \lambda$ is a distinct eigenvalue of $B$ and $\epsilon \rightarrow 0$. The sequence of iterates $u^{(i)}$ converges to an eigenvector associated with an eigenvalue closest to $\hat{\lambda}$. Usually, the starting vector $u^{(0)}$ is chosen to be the normalized vector $(1,1, \ldots, 1)^{T}$. Moreover, if this particular eigenvalue $\lambda$ is known exactly, this method converges in a single iteration [9].

Proposition II.1: Let $\Lambda$ be the set of eigenvalues of the minimal-memory BFGS matrix $B_{k+1}$. Then, for any $\lambda \in$ $\mathbb{R} \backslash \Lambda$, the inverse of $\left(B_{k+1}+\lambda I\right)$ has the general form

$$
\left(B_{k+1}+\lambda I\right)^{-1}=\frac{B_{k+1}^{2}-\left[\beta^{\prime}(\lambda)-\lambda\right] B_{k+1}+\beta(\lambda) I}{\left(\lambda+\theta_{k+1}\right)\left(\lambda^{2}+\beta_{1} \lambda+\beta_{2}\right)},
$$

where $\beta(\lambda)=\left(\lambda+\beta_{1}\right)\left(\lambda+\theta_{k+1}\right)+\beta_{2}$.

Proof: The addition of the term $\lambda I$ on $B_{k+1}$ results that the eigenvalues of $B_{k+1}+\lambda I$ are $x_{i}=\lambda_{i}+\lambda$, where $\lambda_{i}, i=1, \ldots, n$ are the eigenvalues of $B_{k+1}$. Using similar arguments as in proof of Theorem II.1, we have that the characteristic polynomial of $B_{k+1}+\lambda I$ is expressed as follows:

$$
q(x ; \lambda)=\frac{x^{2}-\left(\beta_{1}+2 \lambda\right) x+\lambda^{2}+\beta_{1} \lambda+\beta_{2}}{\left[x-\left(\lambda+\theta_{k+1}\right)\right]^{2-n}} .
$$

Hence, the minimal characteristic polynomial is 


$$
q_{m}(x ; \lambda)=\frac{x^{2}-\left(\beta_{1}+2 \lambda\right) x+\lambda^{2}+\beta_{1} \lambda+\beta_{2}}{\left[x-\left(\theta_{k+1}+\lambda\right)\right]^{-1}} .
$$

Applying the Caley-Hamilton theorem on $B_{k+1}+\lambda I$, we have that $q_{m}\left(B_{k+1}+\lambda I ; \lambda\right)=0$, which yields,

$$
\begin{aligned}
{\left[B_{k+1}+\theta_{k+1} I\right]\left[\left(B_{k+1}+\lambda I\right)^{2}\right.} & -\left(\beta_{1}+2 \lambda\right)\left(B_{k+1}+\lambda I\right) \\
& \left.+\lambda^{2}+\beta_{1} \lambda+\beta_{2} I\right]=0 .
\end{aligned}
$$

Multiplying both sides of the above equation by $\left(B_{k+1}+\lambda I\right)^{-1}$, we yield

$$
\left(B_{k+1}+\lambda I\right)^{-1}=\frac{1}{\left(\theta_{k+1}+\lambda\right)\left(\lambda^{2}+\beta_{1} \lambda+\beta_{2}\right)} .
$$

$\left\{B_{k+1}^{2}-\left(\lambda+\beta_{1}+\theta_{k+1}\right) B_{k+1}+\left[\left(\lambda+\beta_{1}\right)\left(\lambda+\theta_{k+1}\right)+\beta_{2}\right] I\right\}$

By setting $\beta(\lambda)=\left(\lambda+\beta_{1}\right)\left(\lambda+\theta_{k+1}\right)+\beta_{2}$, we obtain relation (13).

Using the above Proposition, along with inverse iteration, we have that the eigenvectors corresponding to distinct eigenvalues of $B_{k+1}$, are the normalized vectors

$$
u(\hat{\lambda})=\frac{B_{k+1}^{2} u-\left[\beta^{\prime}(\hat{\lambda})-\hat{\lambda}\right] B_{k+1} u+\beta(\hat{\lambda}) u}{\left(\hat{\lambda}+\theta_{k+1}\right)\left(\hat{\lambda}^{2}+\beta_{1} \hat{\lambda}+\beta_{2}\right)}
$$

where $\hat{\lambda}=-\lambda+\epsilon$ is a perturbed distinct eigenvalue of $B_{k+1}$ with opposite sign, and $u$ is the unit vector $\frac{1}{\sqrt{n}}(1,1, \ldots, 1)^{T}$. The vectors $B_{k+1} u$ and $B_{k+1}^{2} u$ can be obtained by the iterative form

$$
B_{k+1} v_{i}=\theta_{k+1} v_{i}-\theta_{k+1} \frac{s_{k}^{T} v_{i}}{s_{k}^{T} s_{k}}+\frac{y_{k}^{T} v_{i}}{s_{k}^{T} y_{k}} \quad i=0,1,
$$

with $v_{0}=u$. Using relation (15), after some algebraic computations, the normalized vectors (14) can be expressed by the relation

$$
u(\hat{\lambda})=\frac{c_{u}(\hat{\lambda}) u+c_{s}(\hat{\lambda}) s_{k}+c_{y}(\hat{\lambda}) y_{k}}{\left(\hat{\lambda}^{2}+\beta_{1} \hat{\lambda}+\beta_{2}\right)\left(\hat{\lambda}+\theta_{k+1}\right)},
$$

where the coefficients of $u, s_{k}$ and $y_{k}$ are defined as follows:

$$
\begin{aligned}
& c_{u}(\hat{\lambda})=\hat{\lambda}^{2}+\beta_{1} \hat{\lambda}+\beta_{2} \\
& c_{s}(\hat{\lambda})=\left[\left(\hat{\lambda}+\beta_{1}\right) s_{k}^{T} u-y_{k}^{T} u\right] \frac{\theta_{k+1}}{s_{k}^{T} s_{k}} \quad \text { and } \\
& c_{y}(\hat{\lambda})=-\frac{y_{k}^{T} u}{s_{k}^{T} y_{k}} \hat{\lambda}-\frac{s_{k}^{T} u}{s_{k}^{T} s_{k}} \theta_{k+1}
\end{aligned}
$$

\section{Solving the TRS USing the Minimal-Memory BFGS METHOD}

In this section we apply the results of Section II for solving the large scale trust-region subproblem (1). A global solution to the TRS (1) is characterized by the following well known theorem (Gay [5], Sorensen [19], Moré \& Sorensen [12]):

Theorem III.1: A feasible vector $d^{*}$ is a solution to (1) with corresponding Lagrange multiplier $\lambda^{*}$ if and only if $d^{*}, \lambda^{*}$ satisfy $\left(B+\lambda^{*} I\right) d^{*}=-g$, where $B+\lambda^{*} I$ is positive semi-definite, $\lambda^{*} \geq 0$, and $\lambda^{*}\left(\Delta-\left\|d^{*}\right\|\right)=0$.

From the above Theorem we can distinguish two cases, the standard and the hard case. In the standard case, the optimal non-negative Lagrange multiplier $\lambda^{*}$ belongs to the open interval $\left(-\lambda_{1}, \infty\right)$. When $\lambda^{*} \neq 0$, the TRS (1) has a solution on the boundary of its constraint set, i.e., $\left\|d^{*}\right\|=\Delta$. In this case, the given $n$-dimensional constrained optimization problem is reduced into a zerofinding problem in a single scalar variable $\lambda$, namely, $\phi(\lambda) \equiv 1 / \Delta-1 /\|d(\lambda)\|=0$ (secular equation), that exploits the rational structure of $\|d(\lambda)\|^{2}$. The solution $\lambda$ of the secular equation is based on Newton's method,

$$
\lambda^{\ell+1}=\lambda^{\ell}+\frac{\|d(\lambda)\|}{\|d(\lambda)\|^{\prime}}\left(\frac{\Delta-\|d(\lambda)\|}{\Delta}\right), \quad \ell=0,1,2, \ldots
$$

In Newton's iteration (20) a safeguarding is required to ensure that a solution is found. The safeguarding depends on the fact that $\phi$ is convex and strictly decreasing in $\left(-\lambda_{1}, \infty\right)$. It ensures that $-\lambda_{1} \leq \lambda^{\ell}$, and therefore $B+\lambda^{\ell} I$ is always semi-positive definite [12].

The hard case occurs when $B$ is indefinite and $g$ is orthogonal to every eigenvector corresponding to the most negative eigenvalue $\lambda_{1}$ of the matrix. In this case, there is no $\lambda \in\left(-\lambda_{1}, \infty\right)$ such that $\left\|(B+\lambda I)^{-1} g\right\|=\Delta$. The optimal Lagrange multiplier is $\lambda^{*}=-\lambda_{1}$, and a direction of negative curvature must be produced in order to ensure that $\|d\|=\Delta$. Hence, the optimal solution to the TRS is $d^{*}=-\left(B-\lambda_{1} I\right)^{\dagger} g+\tau u$, where $\tau \in \mathbb{R}$ is such that $\left\|-\left(B-\lambda_{1} I\right)^{\dagger} g+\tau u\right\|=\Delta$ and $u$ is a normalized eigenvector corresponding to $\lambda_{1}$. Moré and Sorensen [12] have showed that the choice of $\tau$ that ensures $\|d\|=\Delta$, is

$$
\tau=\frac{\Delta^{2}-\|p\|^{2}}{p^{T} u_{1}+\operatorname{sgn}\left(p^{T} u\right) \sqrt{\left(p^{T} u\right)^{2}+\Delta^{2}-\|p\|^{2}}},
$$

where $p=-\left(B-\lambda_{1} I\right)^{\dagger} g$, where the symbol ${ }^{\dagger}$ denotes the Moore-Penrose generalized inverse of the matrix.

The above analysis indicates that for been able to solve the TRS (1), we should compute the smallest eigenvalue $\lambda_{1}$ of $B$, the inverse of $B+\lambda I$ for computing the trial step $d(\lambda)$, and, in the hard case, the unit eigenvector $u$ corresponding to $\lambda_{1}$. However, all these quantities required of solving the TRS, have been studied and expressed analytically in the previous section. The following algorithm incorporates both the standard and the hard case and computes a nearly exact solution of the subproblem (1), without using the Cholesky factorization. Moreover, the knowledge of the extreme eigenvalues, results in a straightforward safeguarding procedure for $\lambda$.

Algorithm III.1: (Computation of the trial step)

Step 1: Compute the eigenvalues $\lambda_{i}$ of $B$; given $\epsilon \rightarrow$ $0^{+}$, set the bounds $\lambda_{L}:=\max \left(0,-\lambda_{1}+\epsilon\right)$ and $\lambda_{U}:=$ $\max \left|\lambda_{i}\right|+(1+\epsilon)\|g\| / \Delta$.

Step 2: If $\lambda_{1}>0$, then initialize $\lambda$ by setting $\lambda:=0$ and compute $d(\lambda)$; if $\|d\| \leq \Delta$ stop; else go to Step 4 .

Step 3: Initialize $\lambda$ by setting $\lambda:=-\lambda_{1}+\epsilon$ such that $B+\lambda I$ is positive definite and compute $d(\lambda)$;

a. if $\|d\|>\Delta$ go to Step 4 ;

b. if $\|d\|=\Delta$ stop;

c. if $\|d\|<\Delta$ compute $\tau$ and $u_{1}$ such that $\|-(B+$ $\hat{\lambda} I) g+\tau u_{1} \|=\Delta$; set $d:=d+\tau u_{1}$ and stop; 
Step 4: Use Newton's method to find $\lambda \in\left[\lambda_{L}, \lambda_{U}\right]$ and compute $d(\lambda)$;

Step 5: If $\|d\| \leq \Delta$ stop; else update $\lambda_{L}$ and $\lambda_{U}$ such that $\lambda_{L} \leq \lambda \leq \lambda_{U}$ and go to Step 4 .

The safeguarding scheme required for Newton's iteration (20) uses the parameters $\lambda_{L}$ and $\lambda_{U}$ such that $\left[\lambda_{L}, \lambda_{U}\right]$ is an interval of uncertainty which contains the optimal $\lambda^{*}$. The bounds $\lambda_{L}$ and $\lambda_{U}$ used in Algorithm III.1 have been proposed by Nocedal and Yuan [14]. Clearly, the lower bound $\lambda_{L}$ is greater than $-\lambda_{1}$, which ensures that $B+\lambda I$ is always positive definite.

The eigenvalues in Step 1 of Algorithm III.1 can be computed by means of the characteristic polynomial (4). In Steps 2 and 3 , the computation of the trial step $d(\lambda)$ is based on Proposition II.1. Using relations (15), the trial step can be obtained by the formula

where

$$
d(\lambda)=-\frac{c_{g}(\lambda) g+c_{s}(\lambda) s_{k}+c_{y}(\lambda) y_{k}}{\left(\lambda^{2}+\beta_{1} \lambda+\beta_{2}\right)\left(\lambda+\theta_{k+1}\right)},
$$

$$
\begin{aligned}
& c_{u}(\lambda)=\lambda^{2}+\beta_{1} \lambda+\beta_{2} \\
& c_{s}(\lambda)=\left[\left(\lambda+\beta_{1}\right) s_{k}^{T} g_{k+1}-y_{k}^{T} g_{k+1}\right] \frac{\theta_{k+1}}{s_{k}^{T} s_{k}} \quad \text { and } \\
& c_{y}(\lambda)=-\frac{y_{k}^{T} g_{k+1}}{s_{k}^{T} y_{k}} \lambda-\frac{s_{k}^{T} g_{k+1}}{s_{k}^{T} s_{k}} \theta_{k+1}
\end{aligned}
$$

In Step 3(c) the computation of $\tau$ is obtained from Eq. (21). If the vectors $s_{k}$ and $y_{k}$ are linearly independent, then $u$ is computed by relation (16). In different case, $u$ equals the normalized vector $s_{k}$. In Steps 4 and 5, for computing the Lagrange multiplier $\lambda$ and updating the interval $\left[\lambda_{L}, \lambda_{U}\right]$ we follow the ideas described in [12]. In Newton's method (20), the quantity $\left\|d^{\prime}(\lambda)\right\|$ equals $\|d(\lambda)\|^{\prime}=$ $-d(\lambda)^{T}(B+\lambda I)^{-1} d(\lambda) /\|d(\lambda)\|$. Hence, relation (20) becomes

$\lambda^{\ell+1}=\lambda^{\ell}+\frac{\left\|d_{k+1}(\lambda)\right\|^{2}}{d(\lambda)^{T}\left(B_{k+1}+\lambda I\right)^{-1} d(\lambda)}\left(\frac{\left\|d_{k+1}(\lambda)\right\|-\Delta}{\Delta}\right)$,

for $\ell=0,1,2, \ldots$.

Denoting by $\Pi=d(\lambda)^{T}\left(B_{k+1}+\lambda I\right)^{-1} d(\lambda)$ the denominator in (23), taking into account that $B_{k+1} d_{k+1}(\lambda)=$ $-\left[\lambda d_{k+1}(\lambda)+g_{k+1}\right]$ holds, and using Proposition II.1 we yield

$$
\begin{aligned}
\Pi= & \frac{\left[\beta(\lambda)+\lambda \beta^{\prime}(\lambda)\right]\left\|d(\lambda)_{k+1}\right\|^{2}}{\left(\lambda+\theta_{k+1}\right)\left(\lambda^{2}+\beta_{1} \lambda+\beta_{2}\right)}+ \\
& +\frac{\left[\beta^{\prime}(\lambda)+\lambda\right] d(\lambda)_{k+1}^{T} g_{k+1}+\left\|g_{k+1}\right\|^{2}}{\left(\lambda+\theta_{k+1}\right)\left(\lambda^{2}+\beta_{1} \lambda+\beta_{2}\right)} .
\end{aligned}
$$

If $s_{k}$ and $y_{k}$ are collinear, i.e., relation $y_{k}=\kappa s_{k}$ holds, then relations (22) and (24) are reduced to

$$
d_{k+1}(\lambda)=-\frac{1}{\left(\lambda+\theta_{k+1}\right)} g_{k+1}+\frac{\left(\kappa-\theta_{k+1}\right) s_{k}^{T} g_{k+1}}{(\lambda+\kappa)\left(\lambda+\theta_{k+1}\right) s_{k}^{T} s_{k}} s_{k},
$$

and

$$
\Pi=\frac{\left(2 \lambda+\beta_{1}\right)\|d(\lambda)\|_{k+1}^{2}+d(\lambda)_{k+1}^{T} g_{k+1}}{\lambda^{2}+\beta_{1} \lambda+\beta_{2}},
$$

respectively.

\section{NumERICAL EXPERIMENTS AND ANALYSIS}

For illustrating the behavior of the proposed method in both the standard and the hard case, we use randomly generated TRS instances with dimensions from 100 up to 1000000 variables. The experiments are consisted of medium-size problems with dimensions $n=100,500,1000$, and by larger-size problems $\left(n=10^{4}, 10^{5}, 10^{6}\right)$. For each dimension $n, 1000$ random instances of TRS were generated as follows. The coordinates of the vectors $g, s$, and $y$ were chosen independently from uniform distributions in the interval $\left(-10^{2},+10^{2}\right)$. For the numerical testing we implemented Algorithm III.1 (MLBFGS), using FORTRAN 90 and all numerical experiments were performed on a Pentium $1.86 \mathrm{GHz}$ personal computer with $2 \mathrm{~GB}$ of RAM running Linux operating system.

We compared our method with the GQTPAR [1], and the GLTR [8] algorithm. The GQTPAR algorithm is the subroutine GQTPAR.f from the MINPACK package, and is based on the ideas described in Moré and Sorensen [12] for computing a nearly exact solution of (1). The method uses the Cholesky factorization for the evaluations of the derivatives and the Newton step for $\lambda$, and for the safeguarding and updating of the Lagrange multiplier. The GLTR algorithm proposed by Gould et al. [6], is available as the FORTRAN 90 module HSL_VF05 in the Harwell Subroutine Library. This method is based on a Lanczos tridiagonalization of the matrix $B$ and on the solution of a sequence of problems restricted to Krylov subspaces of $\mathbb{R}^{n}$. It is an alternative to the Steihaug-Toint algorithm [20], [21] and it computes an approximate solution of (1). The algorithm requires only matrix-vector multiplications while it exploits the sparsity of $B$.

Note that the same set of random instances was used throughout for each algorithm. We consider a TRS instance successfully solved, if a solution satisfying $\|(B+\lambda I) d-g\| \leq 10^{-5}$ was computed for both the standard and the hard case. The maximum number of Newton's iterations allowed was 200 and the trust region radius was fixed as $\Delta=10$. For the dimensions $10^{4}, 10^{5}$ and $10^{6}$, results are reported only for MLBFGS and GLTR algorithms, due to the storage requirements of GQTPAR for factoring $B$. Moreover, the GLTR algorithm is not reported in the comparison results for the hard case, since it computes an approximate solution and not a nearly exact solution of the TRS.

To show both the efficacy and accuracy of our method, we have used the performance profile proposed by Dolan and Moré [4]. The performance profile plots the fraction of problems for which any given method is within a factor of the best time. The left axis of the plot shows the percentage of the problems for which a method is the fastest (efficiency). The right side of the plot gives the percentage of the problems that were successfully solved by each of the methods (robustness). The performance measures that have been used are Newton's iterations, solution accuracy and CPU time in seconds. 


\section{A. Random experiments in the standard case}

In the sequel, we present the behavior of the proposed method in the standard case. For each dimension we have consider the following cases:

(a) $s_{k}, y_{k}$ are linearly independent and $\theta_{k+1}=1$;

(b) $s_{k}, y_{k}$ are linearly independent and

$\theta_{k+1}=\left(y_{k}^{T} y_{k}\right) /\left(s_{k}^{T} y_{k}\right)$;

(c) $s_{k}, y_{k}$ are collinear and $\theta_{k+1}=1$;

(d) $s_{k}, y_{k}$ are collinear and $\theta_{k+1}=\left(y_{k}^{T} y_{k}\right) /\left(s_{k}^{T} y_{k}\right)$.

As a total we have 12000 medium size experiments $(n=$ 100, 500, and 1000) and another 12000 large size experiments $\left(n=10^{4}, 10^{5}\right.$, and $\left.10^{6}\right)$.

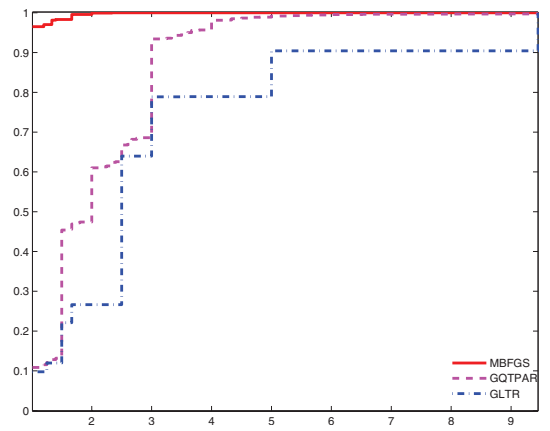

(a) Performance based on number of iterations

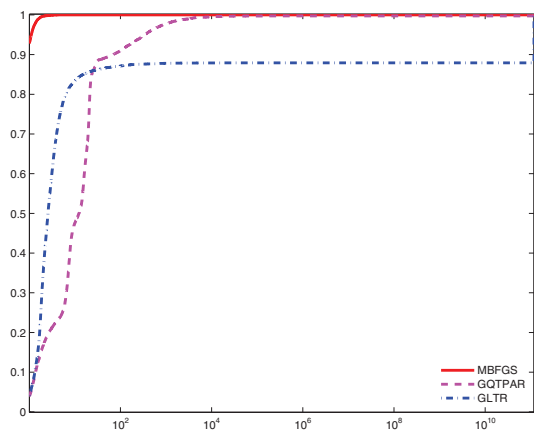

(b) Performance based on accuracy

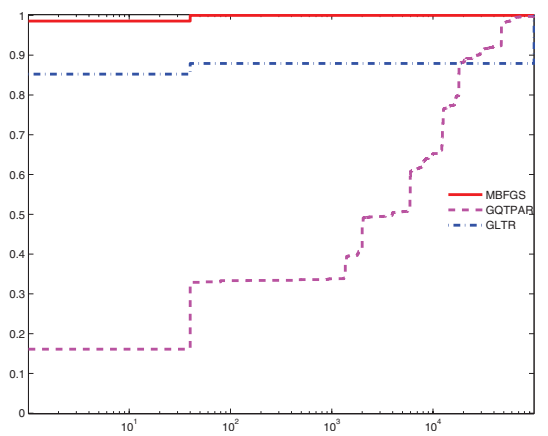

(c) Performance based on CPU time

Fig. 1. Performance profiles for MBFGS, GQTPAR and GLTR on the set of 12000 medium size experiments $(n=100,500$, and 1000).

In Figures 1 and 2 are presented the performance profiles based on Newton iterations, solution accuracy and $\mathrm{CPU}$ time (in seconds) for the medium and large size ex- periments, respectively. In Figure 2 the GQTPAR method was omitted from the comparisons due to insufficient memory limitations. In both figures it is interesting to observe that the best performance regarding all performance metrics was obtained by MBFGS since it is the most robust and efficient method. It is worth noticing that the proposed method significantly outperforms the other two methods in all dimensions.

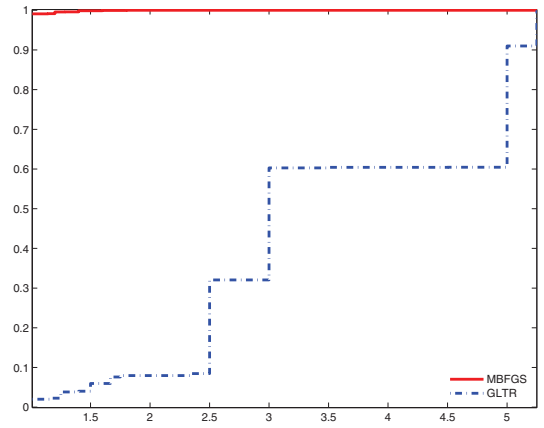

(a) Performance based on number of iterations

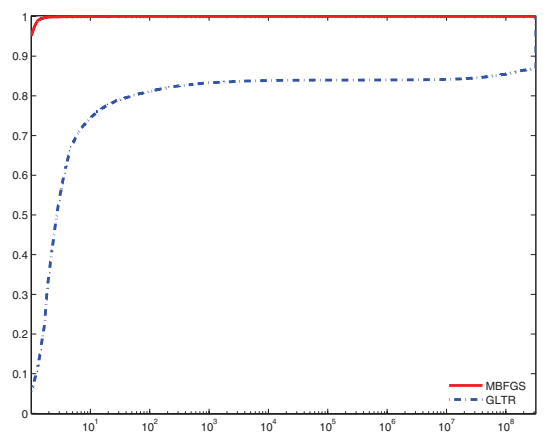

(b) Performance based on accuracy

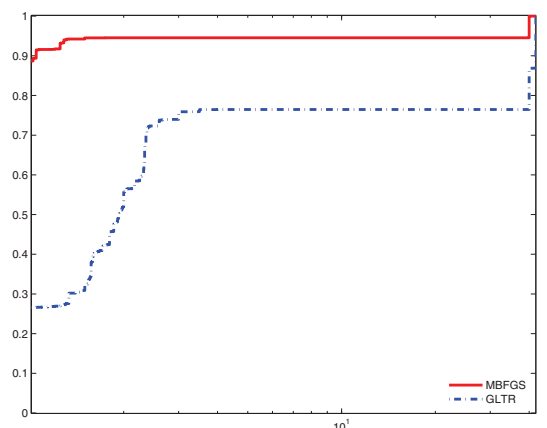

(c) Performance based on CPU time

Fig. 2. Performance profiles for MBFGS and GLTR on the set of 12000 large size experiments $\left(n=10^{4}, 10^{5}\right.$, and $\left.10^{6}\right)$.

\section{B. Random experiments in the hard case}

In order to create instances for the hard case, Matlab's eigs routine was used to compute the smallest eigenvalue $\lambda_{1}$ and the corresponding eigenvector $u$ of the reconstructed matrix $B$ from the vector pairs. We initialized the trust-region radius by $\Delta=10.0 \Delta_{h c}$, where 
$\Delta_{h c}=\left\|\left(B-\lambda_{1} I\right)^{\dagger} g\right\|$ while the vector of the gradient was computed as $g=(-u(n) / u(1), 0, \ldots, 0,1)^{T}$. For each dimension we have consider only the cases (a), (b) and (c) due to the fact that the case (d) occurs only when $g=0$. Totally, we have run 9000 medium size experiments $(n=100,500$, and 1000). In Figure 3 reports the per-

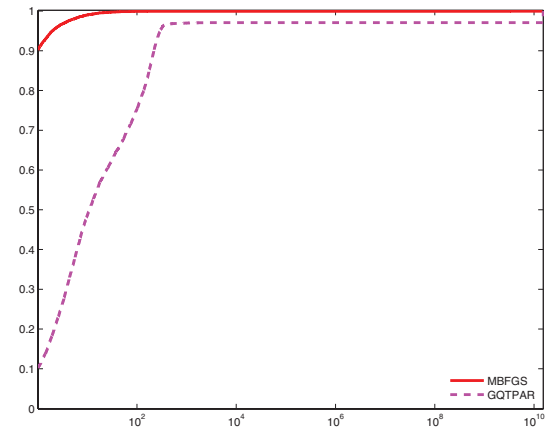

(a) Performance based on accuracy

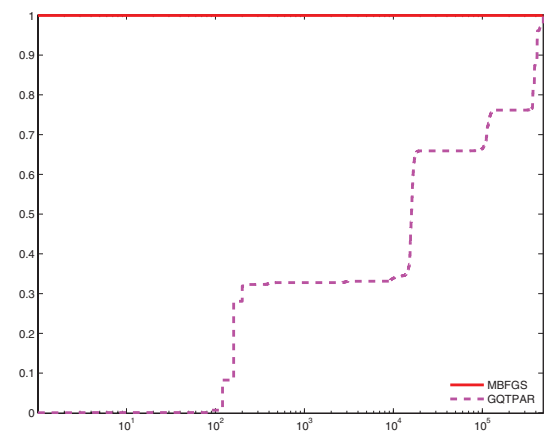

(b) Performance based on CPU time

Fig. 3. Performance profiles for MBFGS and GQTPAR on the set of 9000 medium size experiments $(n=100,500$, and 1000).

formance profiles regarding MBFGS and GQTPAR in terms of solution accuracy and CPU time (in seconds) for medium size problems. The iteration performance metric is not reported since the method MBFGS does not require any Newton's iterations for solving the problem. Obviously, the proposed methods exhibits top performance relative to all performance metrics.

\section{Conclusions}

We have studied the eigenstructure of the minimal memory BFGS matrices, in the general case where the initial matrix is any scaled identity matrix. Our theoretical results have been applied for the solution of the trust region subproblem. Based on the fact that the eigenvalues can immediately be computed with high accuracy, the inverse of $B+\lambda I$ can be expressed in a closed form, and consequently the Cholesky factorization can be completely avoided, the proposed method can solve inexpensively large scale subproblems. The numerical experiments have shown that the proposed method can handle easily both the standard and the hard case, while it provides solutions with high accuracy and small running time, since the amount of memory needed is negligible.

\section{REFERENCES}

[1] E. Anderson, Z. Bai, C. Bischof, J. Demmel, J. Dongarra, J. DuCroz, A. Greenbaum, S. Hammarling, A. McKenney, S. Ostrouchov, and D. Sorensen. LAPACK User's Guide. SIAM, Philadelphia, PA, USA, 1994.

[2] E.G. Birgin and J.M. Martínez. Structured minimal-memory inexact quasi-newton method and secant preconditioners for augmented lagrangian optimization. Comput. Optim. Appl., 39(1):1-16, 2008.

[3] A.R. Conn, N.I.M. Gould, and Ph.L. Toint. Trust-Region Methods. SIAM, Philadelphia, PA, USA, 2000.

[4] E. Dolan and J.J. Moré. Benchmarking optimization software with performance profiles. Mathematical Programming, 91:201213, 2002.

[5] D.M. Gay. Computing optimal locally constrained steps. SIAM J. Sci. Stat. Comput., 2(2):186-197, 1981.

[6] N.I.M. Gould, S. Lucidi, M. Roma, and Ph.L. Toint. Solving the trust-region subproblem using the Lanczos method. SIAM J. Optim., 9(2):504-525, 1999.

[7] W.W. Hager and Y. Krylyuk. Graph partitioning and continuous quadratic programming. SIAM J. Discret. Math., 12(4):500-523, 1999.

[8] HSL 2007. A collection of Fortran codes for large scale scientific computation. 2007. Available from: www.hsl.rl.ac.uk.

[9] I.C.F. Ipsen. Computing an eigenvector with inverse iteration. SIAM Review, 39(2):254-291, June 1997.

[10] D.C. Liu and J. Nocedal. On the limited memory BFGS method for large scale optimization. Math. Program., 45(3):503-528, 1989.

[11] W. Menke. Geophysical Data Analysis: Discrete Inverse Theory. Academic Press, San Diego, 1989.

[12] J.J. Moré and D.C. Sorensen. Computing a trust region step. SIAM J. Sci. Stat. Comput., 4(3):553-572, 1983.

[13] J. Nocedal. Updating quasi-Newton matrices with limited storage. Math. Comput., 35(151):773-782, 1980.

[14] J. Nocedal and Y. Yuan. Combining trust region and line search techniques. In Y. Yuan, editor, Advances in Nonlinear Programming, pages 153-175. Kluwer, Dordrecht, The Netherlands, 1998.

[15] A. Ouorou. Implementing a proximal algorithm for some nonlinear multicommodity flow problems. Networks, 49(1):18-27, 2007.

[16] M.J.D. Powell. A new algorithm for unconstrained optimization. In J.B. Rosen, O.L. Mangasarian, and K. Ritter, editors, Nonlinear Programming, pages 31-65. Academic Press, New York, NY, 1970.

[17] M. Rojas, S.A. Santos, and D.C. Sorensen. A new matrix-free algorithm for the large-scale trust-region subproblem. SIAM J. Optim., 11(3):611-646, 2000.

[18] G.A. Shuldz, R.B. Schnabel, and R.H. Byrd. A family of trustregion-based algorithms for unconstrained minimization with strong global convergence properties. SIAM J. Numer. Anal., 22(1):47-67, February 1985.

[19] D.C. Sorensen. Newton's method with a model trust region modification. SIAM J. Numer. Anal., 19(2):409-426, 1982.

[20] T. Steihaug. The conjugate gradient method and trust regions in large scale optimization. SIAM J. Numer. Anal., 20(3):626637, 1983.

[21] Ph.L. Toint. Towards an efficient sparsity exploiting newton method for minimization. In I.S. Duff, editor, Sparse Matrices and Their Uses, pages 57-87. Academic Press, Inc., New York, NY, 1981.

[22] J.H. Wilkinson. The algebraic eigenvalue problem. Oxford University Press, London, 1965. 